\title{
Calculation of the stability radius of an optimal line balance
}

\author{
Yuri N. Sotskov* Frank Werner ${ }^{* *}$ Aksana Zatsiupa ${ }^{* * *}$ \\ * United Institute of Informatics Problems, Minsk, 220012 Belarus \\ (Tel: 217-375-2842120; e-mail: sotskov@newman.bas-net.by). \\ ** Otto-von-Guericke-Universität, Magdeburg, 39106 Germany \\ (Tel: +493916712025; e-mail: frank.werner@ovgu.de). \\ *** United Institute of Informatics Problems, Minsk, 220012 Belarus \\ (Tel: 217-375-2842125; e-mail: ztp.oksana100@yandex.ru).
}

\begin{abstract}
For an assembly line, it is necessary to minimize the cycle time for processing a partially ordered set of operations $V=\{1, \ldots, n\}$ on a set of $m$ linearly ordered working stations. The number $m$ of stations and the initial processing times $t=\left(t_{1}, \ldots, t_{n}\right)$ of the operations $V$ are given. However, for a subset $\widetilde{V} \subseteq V$ of the manual operations $j \in \widetilde{V}$, it is impossible to fix the processing times $t_{j}$ for the whole life cycle of the assembly line. On the other hand, for each automated operation $i \in V \backslash \widetilde{V}$, the processing time $t_{i}$ is fixed. We investigate the stability of an optimal line balance $b_{0}$ of the assembly line with respect to variations of the processing times $t_{j}, j \in \widetilde{V}$. It is shown how to calculate the stability radius $\rho_{b_{0}}(t)$ of an optimal line balance $b_{0}$, i.e., the maximal value of simultaneous independent variations of the processing times of the manual operations with keeping the optimality of the line balance $b_{0}$. We survey known results on the stability radius of an optimal line balance for a dual problem which is to minimize the number $m$ of the working stations for the given cycle time.
\end{abstract}

Keywords: Optimal line balance; Stability analysis

\section{INTRODUCTION}

We consider a single-model paced assembly line (see Erel and Sarin (1998); Scholl (1999) for definitions and terminology) which continuously manufactures a homogeneous product in large quantities (a mass production).

An assembly line consists of a sequence of $m$ linearly ordered (working) stations, which are linked by a conveyor belt. The set of operations $V$, which have to be repeatedly processed on the $m$ stations, is fixed. Each operation $i \in V$ is indivisible. A station has to perform the same subset of operations of the set $V$ within a cycle time $c$. All the $m$ stations start simultaneously with the processing of a sequence of the operations, which are assigned to a station, and there is no buffer between sequential stations. The technological factors define a partial order on the set of operations $V$ which is given by a digraph $G=(V, A)$, where $V$ is the vertex set and $A$ is the arc set. The set $V$ includes two specific subsets of operations: the nonempty subset $\widetilde{V}$ includes all the manual operations, and the subset $V \backslash \widetilde{V}$ includes all the automated operations.

The simple assembly line balancing problem is to find an optimal line balance for a given set of $m$ stations, i.e., to find a feasible assignment of the operations $V$ to the $m$ stations in such a way that the cycle time $c$ reaches its minimal value. In Scholl (1999), the abbreviation SALBP2 is used to denote such a problem.
Let $\tilde{V}=\{1, \ldots, \tilde{n}\}$ and $V \backslash \tilde{V}=\{\tilde{n}+1, \ldots, n\}$, where $1 \leq \tilde{n} \leq n$. The vectors of the processing times of the operations are denoted as $\widetilde{t}=\left(t_{1}, \ldots, t_{\tilde{n}}\right), \bar{t}=$ $\left(t_{\tilde{n}+1}, \ldots, t_{n}\right)$, and $t=(\widetilde{t}, \bar{t})=\left(t_{1}, \ldots, t_{n}\right)$. Let the nonempty set $V_{k}^{b_{r}}$ of operations be assigned to station $S_{k}$, $k \in\{1, \ldots, m\}$. The assignment $b_{r}$

$$
V=V_{1}^{b_{r}} \bigcup \ldots \bigcup V_{m}^{b_{r}}
$$

of the operations $V$ to $m$ linearly ordered stations $S_{1}, \ldots, S_{m}$ (where

$$
V_{k}^{b_{r}} \bigcap V_{l}^{b_{u}}=\emptyset
$$

with $1 \leq k<l \leq m$ ) is called a line balance, if the following conditions 1 and 2 hold.

1. The assignment $b_{r}$ does not violate the partial order given by the digraph $G=(V, A)$, i.e., inclusion $(i, j) \in A$ implies that operation $i \in V$ is assigned to station $S_{k}$ and operation $j \in V$ is assigned to station $S_{l}$ in such a way that inequalities $1 \leq k \leq l \leq m$ must hold.

2. The assignment $b_{r}$ has to use all $m$ stations:

$$
V_{k}^{b_{r}} \neq \emptyset, k \in\{1, \ldots, m\} .
$$

The line balance $b_{r}$ is optimal if it provides the minimal cycle time $c$. The cycle time $c=c\left(b_{r}, t\right)$ for the line balance $b_{r}$ with the vector $t$ of the operation processing times is defined as

$$
c\left(b_{r}, t\right)=\max _{k=1} \sum_{i \in V_{k}^{b_{r}}} t_{i} .
$$


Let $B=\left\{b_{0}, \ldots, b_{h}\right\}$ be the set of all line balances. The optimality of the line balance $b_{0} \in B$ with the vector $t$ of the operation processing times is defined by the following condition:

3. $c\left(b_{0}, t\right)=\min \left\{c\left(b_{r}, t\right): b_{r} \in B\right\}$

If $j \in \widetilde{V}$, the processing time $t_{j}$ is a positive real number: $t_{j}>0$. However, the value of the processing time $t_{j}$ can vary during the life cycle of the assembly line and a modified processing time $t_{j}^{\prime}$ can be even equal to zero. A zero processing time $t_{j}^{\prime}=0, t_{j}^{\prime} \neq t_{j}$, means that the manual operation

$$
j \in V_{k}^{b_{r}} \bigcap \tilde{V}:=\widetilde{V}_{k}^{b_{r}}
$$

is processed by an additional worker in parallel with processing other operations assigned to the station $S_{k}$. So, due to an additional worker, the processing of operation $j$ does not increase the total processing time for the station $S_{k}$ in the line balance $b_{r}$ :

$$
\sum_{i \in V_{k}^{b_{r}}} t_{i}^{\prime}=\sum_{i \in V_{k}^{b_{r}} \backslash\{j\}} t_{i}^{\prime} .
$$

It is clear that equality (1) is only possible if $t_{j}^{\prime}=0$.

If $j \in \widetilde{V}$, the processing time $t_{j}$ is a real number which is fixed during the whole life cycle of the assembly line. One can assume that the strict inequality $t_{i}>0$ holds for each automated operation $i \in V \backslash \widetilde{V}$ since an operation with a fixed zero processing time has no influence on a solution of the problem SALBP-2.

In contrast to a stochastic assembly line balancing problem (see the survey Erel and Sarin (1998)), we do not assume any probability distribution known in advance for the random processing time $t_{j} \in \widetilde{V}$.

Moreover, this paper does not deal with an algorithm for constructing an optimal line balance. It is assumed that an optimal line balance $b_{0} \in B$ is somehow already constructed for the initial vector $t=\left(t_{1}, \ldots, t_{n}\right)$ of the operation processing times. Our aim is to investigate the stability of the optimality of a line balance $b_{0}$ with respect to simultaneous independent variations of the processing times of the manual operations $\widetilde{V}$.

In what follows, we show how to calculate the stability radius of an optimal line balance $b_{0} \in B$. The stability radius may be interpreted as the maximum of simultaneous independent variations of the processing times of the manual operations $\widetilde{V}$ with definitely keeping the optimality of line balance $b_{0}$.

It should be noted that the processing times $t_{i}, i \in V$, are assumed to be real numbers (in contrast to the assumption used by Scholl (1999) that the processing times must be integers).

\section{DEFINITION OF THE STABILITY RADIUS}

The main question under consideration is as follows. How much can the components of a vector $\widetilde{t}$ simultaneously and independently be modified such that the line balance $b_{0}$, which is optimal for the initial vector $t$ of the operation processing times, remains optimal for the modified vector $t^{\prime}$ of the operation processing times?

To be more precise, we study the stability radius of an optimal line balance, which is defined similarly to the stability radius of an optimal schedule (see Bräsel et al. (1996); Sotskov et al. (1997, 2010)).

If the stability radius of the line balance $b_{0}$ is strictly positive, then any simultaneous independent changes of the operation times $t_{j}, j \in \widetilde{V}$, within the ball with this radius and the center $\widetilde{t}$ definitely keep the optimality of the line balance $b_{0}$.

On the other hand, if the stability radius of the line balance $b_{0}$ is equal to zero, then even small changes of the processing times of the manual operations may deprive the optimality of the line balance $b_{0}$.

Let $R^{\tilde{n}}$ denote the space of real vectors $\tilde{t}=\left(t_{1}, \ldots, t_{\tilde{n}}\right)$ with the Chebyshev metric, i.e., the distance $d\left(\widetilde{t}, \widetilde{t^{\prime}}\right)$ between the vector $\widetilde{t}$ and the vector $\widetilde{t}^{\prime}=\left(t_{1}^{\prime}, \ldots, t_{\tilde{n}}^{\prime}\right)$ is defined as follows:

$$
d\left(\widetilde{t}, \widetilde{t}^{\prime}\right)=\max \left\{\left|t_{i}-t_{i}^{\prime}\right|: i \in \widetilde{V}\right\},
$$

where $\left|t_{i}-t_{i}^{\prime}\right|$ denotes the absolute value of the difference $t_{i}-t_{i}^{\prime}$. Let $B(t)$ be the set of all line balances in the set $B$, which are optimal for the vector $t$ of the operation processing times. $R_{+}^{\tilde{n}}$ denotes the space of non-negative real vectors.

Definition 1. The closed ball $O_{\rho}(\widetilde{t})$ in the space $R^{\tilde{n}}$ with the radius $\rho \in R_{+}^{1}$ and the center $\widetilde{t} \in R_{+}^{\tilde{n}}$ is called a stability ball of the line balance $b_{0} \in B(t)$, if for each vector $t^{\prime}=\left(\widetilde{t^{\prime}}, \bar{t}\right)$ of the processing times with

$$
\widetilde{t}^{\prime} \in O_{\rho}(\widetilde{t}) \bigcap R_{+}^{\tilde{n}}
$$

the line balance $b_{0}$ remains optimal. The maximal value $\rho_{b_{0}}(t)$ of the radius $\rho$ of a stability ball $O_{\rho}(\widetilde{t})$ is called the stability radius of the line balance $b_{0}$.

In Definition 1, the initial vector $t=(\widetilde{t}, \bar{t})$ of the processing times is fixed, while the vector $\widetilde{t}^{\prime}$ of the processing times of the manual operations may vary within the intersection of the closed ball $O_{\rho}(\widetilde{t})$ with the space $R_{+}^{\tilde{n}}$.

Let $W\left(b_{r}, t\right)$ be the set of subsets $\widetilde{V}_{k}^{b_{r}}, k \in\{1, \ldots, m\}$, for which equality (2) holds:

$$
t\left(V_{k}^{b_{r}}\right)=c\left(b_{r}, t\right) .
$$

Hereafter, we use the notation

$$
t\left(V_{k}^{b_{r}}\right)=\sum_{i \in V_{k}^{b_{r}}} t_{i},
$$

where $t$ indicates a vector $t=(\tilde{t}, \bar{t})=\left(t_{1}, \ldots, t_{n}\right) \in R_{+}^{n}$, for which the sum

has been calculated.

$$
\sum_{i \in V_{k}^{b_{r}}} t_{i}
$$

Sotskov et al. (2005) proved the following criterion for a zero value of the stability radius $\rho_{b_{0}}(t)$.

Theorem 1. Let $b_{0} \in B(t)$. The equality $\rho_{b_{0}}(t)=0$ holds if and only if there exists a line balance $b_{s} \in B(t)$ such that condition (3) does not hold: 


$$
W\left(b_{0}, t\right) \subseteq W\left(b_{s}, t\right)
$$

In Section 3, we present a sketch of the proof of Theorem 2 which allows us to calculate the stability radius $\rho_{b_{0}}(t)$ for the line balance $b_{0} \in B(t)$ in the case when $\rho_{b_{0}}(t) \neq 0$.

\section{CALCULATION OF THE STABILITY RADIUS}

Assume that for a line balance $b_{0} \in B(t)$, there does not exist a line balance $b_{s} \in B(t)$ such that inclusion (3) holds. Due to Theorem 1 , the stability radius $\rho_{b_{0}}(t)$ is strictly positive. First, we consider the following case $(\mathrm{j})$.

Case $(\mathrm{j}): B(t)=\left\{b_{0}\right\}$

In order to calculate the exact value of a strictly positive stability radius $\rho_{b_{0}}(t)>0$, we shall look for a line balance $b_{r} \in B \backslash\left\{b_{0}\right\}$ and for a vector $\widetilde{t^{\epsilon}}=\left(t_{1}^{\epsilon}, \ldots, t_{\tilde{n}}^{\epsilon}\right) \in R_{+}^{\tilde{n}}$ such that the following strict inequality holds:

$$
c\left(b_{r}, t^{\epsilon}\right)<c\left(b_{0}, t^{\epsilon}\right)
$$

where $t^{\epsilon}=\left(\widetilde{t^{\epsilon}}, \bar{t}\right)$ and the vector $\widetilde{t^{\epsilon}}$ may be as close as desired to the vector $\widetilde{t}$ provided that the strict inequality (4) holds.

Since the value $c\left(b_{0}, t\right)$ linearly depends on the components of the vector $\tilde{t}=\left(t_{1}, \ldots, t_{\tilde{n}}\right)$, before reaching the strict inequality in (4) via a continuous change of the components of the vector $\widetilde{t}$, one first reaches the following equality

$$
c\left(b_{r}, t^{\prime}\right)=c\left(b_{0}, t^{\prime}\right)
$$

for some vector $\widetilde{t}^{\prime}=\left(t_{1}^{\prime}, \ldots, t_{\tilde{n}}^{\prime}\right)$, for which the optimal line balance $b_{0}$ becomes unstable, i.e., the equality

$$
\rho_{b_{0}}\left(t^{\prime}\right)=0
$$

must hold.

Due to Theorem 1, from equality (5), it follows that there exists a line balance $b_{r} \in B\left(t^{\prime}\right)$ such that the condition $W\left(b_{0}, t^{\prime}\right) \subseteq W\left(b_{r}, t^{\prime}\right)$ does not hold. Hence, one can construct a vector $\widetilde{t^{\epsilon}}$, for which inequality (4) holds and $d\left(\widetilde{t^{\epsilon}}, \widetilde{t}^{\prime}\right)=\epsilon$, where the positive number $\varepsilon \in R_{+}^{1}$ may be as small as desired.

Thus, the calculation of the stability radius $\rho_{b_{0}}(t)$ for the line balance $b_{0} \in B(t)$ may be reduced to the construction of a vector $\widetilde{t}^{\prime} \in R_{+}^{\tilde{n}}$, which is the closest one to the vector $\tilde{t}$ provided that equality (5) holds.

In case $(\mathrm{j})$, we have to compare the line balance $b_{0}$ with the non-optimal line balances $b_{r} \in B \backslash\left\{b_{0}\right\}$.

Let $t\left(V_{k}^{b_{r}}\right)>t\left(V_{u}^{b_{0}}\right)$ and $\left|\widetilde{V}_{u}^{b_{0}} \oplus \widetilde{V}_{k}^{b_{r}}\right| \geq 1$.

Hereafter, the $\operatorname{sign} \oplus$ denotes the direct summation of two sets as follows:

$$
\widetilde{V}_{u}^{b_{0}} \oplus \widetilde{V}_{k}^{b_{r}}=\left\{\widetilde{V}_{u}^{b_{0}} \bigcup \widetilde{V}_{k}^{b_{r}}\right\} \backslash\left\{\widetilde{V}_{u}^{b_{0}} \bigcap \widetilde{V}_{k}^{b_{r}}\right\} .
$$

It is easy to see that one will reach the equality

if the following value

$$
t^{\prime}\left(V_{k}^{b_{r}}\right)=t^{\prime}\left(V_{u}^{b_{0}}\right)
$$

$$
\delta_{b_{0}, u}^{b_{r}, k}=\frac{t\left(V_{k}^{b_{r}}\right)-t\left(V_{u}^{b_{0}}\right)}{\left|\widetilde{V}_{u}^{b_{0}} \oplus \widetilde{V}_{k}^{b_{r}}\right|}
$$

will be added (will be subtracted, respectively) to the processing time $t_{i}$ for each operation $i \in \widetilde{V}_{u}^{b_{o}} \backslash \widetilde{V}_{k}^{b_{r}}$, i.e.,

$$
t_{i}^{\prime}=t_{i}+\delta_{b_{0}, u}^{b_{r}, k}
$$

(from the processing time $t_{j}$ for each operation $j \in \widetilde{V}_{k}^{b_{r}} \backslash$ $\widetilde{V}_{u}^{b_{0}}$, i.e.,

$$
\left.t_{j}^{\prime}=t_{j}-\delta_{b_{0}, u}^{b_{r}, k}\right)
$$

It should be noted that in the latter modification, a component $t_{j}^{\prime}=t_{j}-\delta_{b_{0}, u}^{b_{r}, k}$ of the vector $t^{\prime}=\left(t_{1}^{\prime}, \ldots, t_{n}^{\prime}\right)$ may become negative.

In order to get a non-negative modified vector $t^{\prime}$, one has to test the operations of the set $\widetilde{V}_{k}^{b_{r}} \backslash \widetilde{V}_{u}^{b_{0}}$ in non-decreasing order of their processing times. Let $t_{(0)}^{u k}$ be equal to zero and let

$$
t_{(0)}^{u k}, t_{(1)}^{u k}, \ldots, t_{\left(w_{u k}\right)}^{u k}
$$

denote a non-decreasing sequence of the processing times of the operations from the set $\widetilde{V}_{k}^{b_{r}} \backslash \widetilde{V}_{u}^{b_{0}}$, where $w_{u k}=$ $\left|\widetilde{V}_{k}^{b_{r}} \backslash \widetilde{V}_{u}^{b_{0}}\right|$. A non-negative vector $t^{\prime}$ will be obtained if $\delta_{b_{0}, u}^{b_{r}, k}$ defined in (6) will be substituted by the following value:

$$
\delta_{b_{0}, u}^{b_{r}, k}=\max _{\beta=0, \ldots, w_{u k}} \frac{t\left(V_{k}^{b_{r}}\right)-t\left(V_{0}^{b_{u}}\right)-\sum_{\alpha=0}^{\beta} t_{(\alpha)}^{u k}}{\max \left\{1,\left|\widetilde{V}_{u}^{b_{0}} \oplus \widetilde{V}_{k}^{b_{r}}\right|-\beta\right\}} .
$$

In order to obtain the minimal distance $d\left(t, t^{\prime}\right)$ between the desired vector $t^{\prime}$ and the initial vector $t$, one has to take the following maximum on $k$

$$
\max _{t\left(V_{k}^{b_{r}}\right)>c\left(b_{0}, t\right)} \delta_{b_{0}, u}^{b_{r}, k}
$$

then the following minimum on $u$

$$
\delta\left(b_{0}, b_{r}, t\right)=\min _{\left|\widetilde{V}_{u}^{b_{0}} \oplus \widetilde{V}_{k}^{b_{r}}\right| \geq 1} \max _{t\left(V_{k}^{b_{r}}\right)>c\left(b_{0}, t\right)} \delta_{b_{0}, u}^{b_{r}, k},
$$

and, finally, the following minimum

$$
\delta\left(b_{0}, t\right)=\min _{b_{r} \in B \backslash\left\{b_{0}\right\}} \min _{\left|\widetilde{V}_{u}^{b_{0}} \oplus \widetilde{V}_{k}^{b_{r}}\right| \geq 1} \max _{t\left(V_{k}^{b_{r}}\right)>c\left(b_{0}, t\right)} \delta_{b_{0}, u}^{b_{r}, k} .
$$

Summarizing, we conclude that equality

must hold in the case $(\mathrm{j})$.

$$
\rho_{b_{0}}(t)=\delta\left(b_{0}, t\right)
$$

Next, we consider the remaining possible case (jj).

Case (jj): $B(t) \backslash\left\{b_{0}\right\} \neq \emptyset$

Similarly as in the case (j), we have to calculate the value

$$
\delta\left(b_{0}, t\right)=\min _{b_{r} \in B \backslash B(t)} \min _{\left|\widetilde{V}_{u}^{b_{0}} \oplus \widetilde{V}_{k}^{b_{r}}\right| \geq 1} \max _{t\left(V_{k}^{b_{r}}\right)>c\left(b_{0}, t\right)} \delta_{b_{0}, u}^{b_{r}, k},
$$

where $\delta_{b_{0}, u}^{b_{r}, k}$ is defined in (7) and in contrast to (9), the first minimum is taken for all line balances $b_{r} \in B \backslash B(t)$.

In case $(\mathrm{jj})$, along with calculating $\delta\left(b_{0}, t\right)$, we have to compare the line balance $b_{0}$ with all other optimal line balances $b_{s} \in B(t), b_{s} \neq b_{0}$.

Let $\left|\widetilde{V}_{u}^{b_{0}} \oplus \widetilde{V}_{k}^{b_{s}}\right| \geq 1$ and

$$
t\left(V_{u}^{b_{0}}\right)<c\left(b_{s}, t\right)=t\left(V_{k}^{b_{s}}\right),
$$


where $b_{s} \in B(t) \backslash\left\{b_{0}\right\}$. It is easy to see that one will reach the equality

$$
t^{\prime}\left(V_{k}^{b_{s}}\right)=t^{\prime}\left(V_{u}^{b_{0}}\right)
$$

if the following value

$$
\Delta_{b_{0}, u}^{b_{s}, k}=\frac{c\left(b_{s}, t\right)-t\left(V_{u}^{b_{0}}\right)}{\left|\widetilde{V}_{u}^{b_{0}} \oplus \widetilde{V}_{k}^{b_{s}}\right|}
$$

will be added (will be subtracted, respectively) to the processing time $t_{i}$ for each operation $i \in \widetilde{V}_{u}^{b_{0}} \backslash \widetilde{V}_{k}^{b_{s}}$, i.e.,

$$
t_{i}^{\prime}=t_{i}+\Delta_{b_{0}, u}^{b_{s}, k}
$$

(from the processing time $t_{j}$ for each operation $j \in \widetilde{V}_{k}^{b_{s}}$ ) $\widetilde{V}_{u}^{b_{0}}$, i.e.,

$$
\left.t_{j}^{\prime}=t_{j}-\Delta_{b_{0}, u}^{b_{s}, k}\right)
$$

Let $t_{(0)}^{u k}$ be equal to zero and let

$$
t_{(0)}^{u k}, t_{(1)}^{u k}, \ldots, t_{\left(w_{u k}\right)}^{u k}
$$

denote a non-decreasing sequence of the processing times of the operations from the set $\widetilde{V}_{k}^{b_{s}} \backslash \widetilde{V}_{u}^{b_{0}}$, where $w_{u k}=\left|\widetilde{V}_{k}^{b_{s}}\right\rangle$ $\widetilde{V}_{u}^{b_{0}} \mid$. A non-negative vector $t^{\prime}$ will be obtained if $\Delta_{b_{0}, u}^{b_{s}, k}$ defined in (11) will be substituted by the value $\Delta_{b_{0}, u}^{b_{s}, k}$ defined in (12):

$$
\Delta_{b_{0}, u}^{b_{s}, k}=\max _{\beta=0, \ldots, w_{u k}} \frac{c\left(b_{s}, t\right)-t\left(V_{u}^{b_{0}}\right)-\sum_{\alpha=0}^{\beta} t_{(\alpha)}^{u k}}{\max \left\{1,\left|\widetilde{V}_{u}^{b_{0}} \oplus \widetilde{V}_{k}^{b_{s}}\right|-\beta\right\}} .
$$

In order to obtain the minimal distance $d\left(t, t^{\prime}\right)$ of the desired vector $t^{\prime}$ to the initial vector $t$, one has to take the following maximum on $k$

$$
\max _{t\left(V_{u}^{b_{0}}\right)<c\left(b_{s}, t\right)=t\left(V_{k}^{b_{s}}\right)} \Delta_{b_{0}, u}^{b_{s}, k},
$$

then the following minimum on $u$

$$
\delta\left(b_{0}, b_{s}, t\right)=\min _{\left|\widetilde{V}_{u}^{b_{0}} \oplus \widetilde{V}_{k}^{b_{s}}\right| \geq 1} \max _{t\left(V_{u}^{b_{0}}\right)<c\left(b_{s}, t\right)=t\left(V_{k}^{b_{s}}\right)} \Delta_{b_{0}, u}^{b_{s}, k},
$$

and, finally, the following minimum

$$
\begin{gathered}
\Delta\left(b_{0}, t\right)= \\
\min _{b_{r} \in B \backslash\left\{b_{0}\right\}} \min _{\left|\widetilde{V}_{u}^{b_{0}} \oplus \widetilde{V}_{k}^{b_{s}}\right| \geq 1} \max _{t\left(V_{u}^{b_{0}}\right)<c\left(b_{s}, t\right)=t\left(V_{k}^{b_{s}}\right)} \Delta_{b_{0}, u}^{b_{s}, k} .
\end{gathered}
$$

Summarizing, we conclude that equality

$$
\rho_{b_{0}}(t)=\min \left\{\delta\left(b_{0}, t\right), \Delta\left(b_{0}, t\right)\right\}
$$

must hold in the case $(\mathrm{jj})$. So, for the general case, we can formulate the following theorem.

Theorem 2. If $b_{0} \in B(t)$ and $\rho_{b_{0}}(t) \neq 0$, then

$$
\rho_{b_{0}}(t)=\min \left\{\delta\left(b_{0}, t\right), \Delta\left(b_{0}, t\right)\right\},
$$

where $\Delta\left(b_{0}, t\right)$ is defined in (14) and $\delta\left(b_{0}, t\right)$ is defined either in (9) or in (10).

Because of the page limit, we cannot present the complete proof of Theorem 2 (we are forced to remove some important details in the above argumentation).

The implementation of Theorem 2 is demonstrated on the calculation of the stability radius $\rho_{b_{0}}(t)$ for a small example given in Section 5 .

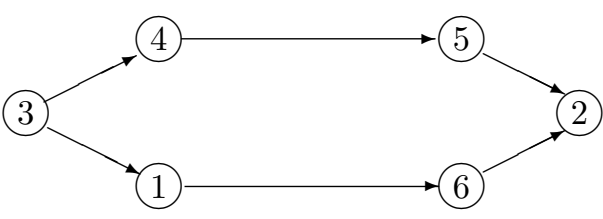

Fig. 1. The digraph $G=(V, A)$ defining a partial order on the set of operations $V$.

In Section 4, we show how one can restrict the number of line balances from the set $B \backslash\left\{b_{0}\right\}$, which have to be compared with the line balance $b_{0}$ for calculating the stability radius $\rho_{b_{0}}(t)$ using Theorem 2 .

\section{REDUNDANT LINE BALANCES FOR CALCULATING THE STABILITY RADIUS}

Due to Theorem 2, the calculation of the stability radius of an optimal line balance is reduced to a time-consuming calculation on the set of line balances $B$. In the worst case, an optimal line balance $b_{0}$ must be compared with all the line balances from the set $B$. In order to restrict the number of line balances $b_{r} \in B \backslash\left\{b_{0}\right\}$ with which a comparison of the line balance $b_{0}$ has to be done for calculating the stability radius $\rho_{b_{0}}(t)>0$, one can use the lower bound on the value $\delta\left(b_{0}, b_{g}, t\right)$ given in Lemma 3 which is presented without a proof.

We denote

$$
\tilde{n}_{b_{r}}=\max \left\{\left|\widetilde{V}_{k}^{b_{r}}\right|: k \in\{1, \ldots, m\}\right\} .
$$

Lemma 3. Let $b_{0} \in B(t)$ and $b_{r} \in B \backslash\left\{b_{0}\right\}$. Then there is no need to compare the line balance $b_{0}$ with the line balance $b_{r}$ if inequality

$$
\delta\left(b_{0}, b_{g}, t\right) \geq \frac{c\left(b_{r}, t\right)-c\left(b_{0}, t\right)}{\min \left\{\tilde{n}, \tilde{n}_{b_{r}}+\tilde{n}_{b_{0}}\right\}}
$$

holds for some line balance $b_{g} \in B \backslash\left\{b_{0}, b_{r}\right\}$.

The value $\delta\left(b_{0}, b_{g}, t\right)$ used in Lemma 3 is defined both in (9) and in (13).

In Lemma 3 , it is assumed that the line balance $b_{0}$ is already compared with the line balance $b_{g}$. As a result, the following upper bound on the stability radius $\rho_{b_{0}}(t)$ is obtained:

$$
\rho_{b_{0}}(t) \leq \delta\left(b_{0}, b_{g}, t\right)
$$

Due to Lemma 3 , the line balance $b_{r} \in B \backslash\left\{b_{0}\right\}$ may make precise the achieved volume of the stability ball $O_{\rho}(\widetilde{t})$ only if inequality (15) does not hold. Thus, the line balances $b_{r} \in B \backslash\left\{b_{0}\right\}$ must be compared with the line balance $b_{0}$ in the order of increasing the right-hand side of inequality (15).

\section{EXAMPLE}

Let $m=3, n=6, \tilde{n}=3$ and $t=(\widetilde{t}, \bar{t})=(4,1,6,3,7,4)$. The set $\widetilde{V}=\{1,2,3\}$ includes all the manual operations. The set $V \backslash \widetilde{V}=\{4,5,6\}$ includes all the automated operations. The digraph $G=(V, A)$ defining a partial order on the set of operations $V$ is presented in Fig. 1.

If all the processing times are integers, then the following lower bound on the minimal cycle time may be used: 


$$
\min \left\{c\left(b_{r}, t\right): b_{r} \in B\right\} \geq\left\lceil\sum_{i=1}^{n} \frac{t_{i}}{m}\right\rceil
$$

where $\lceil a\rceil$ denotes the smallest integer greater than or equal to $a$.

One can convince that the line balance $b_{0}$

$$
V=\{3,4\} \bigcup\{1,6\} \bigcup\{2,5\}
$$

is optimal. Indeed, for the line balance $b_{0}$, we obtain

$$
\left\lceil\sum_{i=1}^{n} \frac{t_{i}}{m}\right\rceil=\left\lceil\sum_{i=1}^{6} \frac{t_{i}}{3}\right\rceil=\left\lceil\frac{26}{3}\right\rceil=9=c\left(b_{0}, t\right),
$$

which implies that $b_{0}$ is an optimal line balance since $c\left(b_{0}, t\right)$ is equal to the right-hand side of inequality (16).

Similarly, one can convince that the line balance $b_{1}$

$$
V=\{3,4\} \bigcup\{5\} \bigcup\{1,2,6\}
$$

is also optimal. The other line balances are non-optimal for the vector $t=(\widetilde{t}, \bar{t})=(4,1,6,3,7,4)$ of the operation processing times.

For the line balance $b_{1}$, there exists a line balance $b_{0} \in B(t)$ such that condition (3) does not hold, namely:

$$
\{\{3\},\{1,2\}\}=W\left(b_{1}, t\right) \nsubseteq W\left(b_{0}, t\right)=\{\{3\}\} .
$$

So, due to Theorem 1 , we obtain the equality

$$
\rho_{b_{1}}(t)=0
$$

which means that the optimality of the line balance $b_{1} \in$ $B(t)$ is unstable: even small changes of the processing times of the manual operations 1,2 and 3 may deprive the optimality of the line balance $b_{1}$.

On the other hand, for the line balance $b_{0}$, we obtain

$$
\{\{3\}\}=W\left(b_{0}, t\right) \subseteq W\left(b_{1}, t\right)=\{\{3\},\{1,2\}\},
$$

where $\left\{b_{1}\right\}=B(t) \backslash\left\{b_{0}\right\}$. So, due to Theorem 1 , the strict inequality

$$
\rho_{b_{0}}(t)>0
$$

must hold, and the optimality of the line balance $b_{0} \in B(t)$ is stable: there exists a closed ball $O_{\rho}(\widetilde{t})$ in the space $R^{3}$ with the positive radius $\rho \in R_{+}^{1}$ and the center $\tilde{t} \in R_{+}^{3}$ such that inclusion $b_{0} \in B\left(t^{\prime}\right)$ holds for any modified vector $t^{\prime}=\left(\widetilde{t^{\prime}}, \bar{t}\right)$ of the operation processing times with

$$
\widetilde{t}^{\prime} \in O_{\rho}(\widetilde{t}) \bigcap R_{+}^{3} .
$$

Due to Theorem 2, we can calculate the maximum value $\rho_{b_{0}}(t)$ of such a radius $\rho$.

First, we compare the line balance $b_{0}$ with the line balance $b_{1}$, which is also optimal for the initial vector $t=$ $(4,1,6,3,7,4): b_{1} \in B(t)$. Using formula (13), we calculate

$$
\delta\left(b_{0}, b_{1}, t\right)=1 \text {. }
$$

Using formula (14), we obtain $\Delta\left(b_{0}, t\right)=1$.

Using bound (15) with the left-hand side $\delta\left(b_{0}, b_{1}, t\right)$ equal to 1 , we can convince that, to calculate the value $\delta\left(b_{0}, t\right)$ given in (9), it is sufficient to compare the line balance $b_{0}$ with five line balances as follows:

with the line balance $b_{2}$

$$
V=\{3,4\} \bigcup\{1,5\} \bigcup\{2,6\},
$$

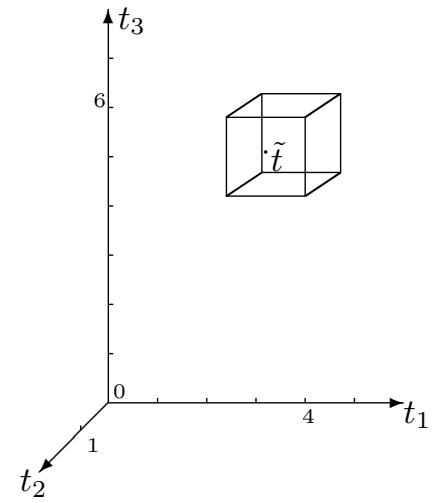

Fig. 2. The stability ball $O_{\rho}(\tilde{t})$ with the radius $\rho=\rho_{b_{0}}(t)=$ $\frac{2}{3}$ and the center $\tilde{t}=\{4,1,6\}$ for the line balance $b_{0} \in B(t)=\left\{b_{0}, b_{1}\right\}$.

with the line balance $b_{3}$

$$
V=\{1,3\} \bigcup\{4,5\} \bigcup\{2,6\},
$$

with the line balance $b_{4}$

$$
V=\{1,3\} \bigcup\{4,6\} \bigcup\{2,5\},
$$

with the line balance $b_{5}$

and with the line balance $b_{6}$

$$
V=\{1,3\} \bigcup\{6\} \bigcup\{2,4,5\},
$$

$$
V=\{3\} \bigcup\{4,5\} \bigcup\{1,2,6\} .
$$

Using formula (8), we calculate

$$
\begin{aligned}
\delta\left(b_{0}, b_{2}, t\right) & =1, \\
\delta\left(b_{0}, b_{3}, t\right) & =1, \\
\delta\left(b_{0}, b_{4}, t\right) & =\frac{2}{3}, \\
\delta\left(b_{0}, b_{5}, t\right) & =\frac{2}{3}, \\
\delta\left(b_{0}, b_{6}, t\right) & =1 .
\end{aligned}
$$

Using formula (9), we obtain

$$
\delta\left(b_{0}, t\right)=\min \left\{1,1, \frac{2}{3}, \frac{2}{3}, 1\right\}=\frac{2}{3} .
$$

Using Theorem 2, we obtain

$$
\rho_{b_{0}}(t)=\min \left\{\delta\left(b_{0}, t\right), \Delta\left(b_{0}, t\right)\right\}=\min \left\{\frac{2}{3}, 1\right\}=\frac{2}{3} .
$$

The stability ball $O_{\rho}(\tilde{t})$ with the radius

$$
\rho=\rho_{b_{0}}(t)=\frac{2}{3}
$$

and the center $\tilde{t}=\{4,1,6\}$ for the line balance $b_{0} \in B(t)$ is presented in Fig. 2.

\section{A SURVEY OF STABILITY RESULTS FOR A DUAL PROBLEM}

At the stage of the design of the assembly line, the following problem, which is dual to the problem SALBP2, has to be solved: minimize the number of stations $m$ for processing a set of partially ordered operations $V=\{1, \ldots, n\}$ within the given cycle time $c$. 
The assignment $b_{r}$

$$
V=V_{1}^{b_{r}} \bigcup \ldots \bigcup V_{m}^{b_{r}}
$$

of the operations $V$ to $m$ linearly ordered stations $S_{1}, \ldots, S_{m}$ (where $V_{k}^{b_{r}} \cap V_{l}^{b_{u}}=\emptyset$ with $1 \leq k<l \leq m$ ) is a feasible line balance, if condition 1 (see Section 1) and the following condition $2^{*}$ hold.

$2^{*}$. The given cycle time $c$ is not violated for each station $S_{k}, k \in\{1, \ldots, m\}$, i.e., the station time $t\left(V_{k}^{b_{r}}\right)$ has to be no greater than the cycle time $c$.

The feasible line balance $b_{0} \in B$ is optimal if it provides the minimal number $m=m_{b_{0}}$ of stations used, i.e., the following condition $3^{*}$ holds.

$3^{*} . m=m_{b_{0}}=\min \left\{m\left(b_{r}\right): b_{r} \in B\right\}$

Scholl (1999) uses the abbreviation SALBP-1 to denote such a dual problem.

In practice, at the design stage of an assembly line, the cycle time $c$ may be calculated on the basis of the customer demands for the finished products, which will be assembled on the designed assembly line.

Similarly to the problem SALBP-2, considered in Sections 1-5, problem SALBP-1 is binary NP-hard even for the case of two stations $(m=2)$ used in the optimal line balance.

A stability analysis for the problem SALBP-1 was developed by Sotskov and Dolgui (2001); Sotskov et al. (2006) with slightly different definitions of the stability radii. In particular, Sotskov et al. (2006) use the following definition.

Definition 2. For the problem SALBP-1, the open ball $O_{\rho}^{1}(\widetilde{t})$ in the space $R^{\tilde{n}}$ with the radius $\rho \in R_{+}^{1}$ and the center $\tilde{t} \in R_{+}^{\tilde{n}}$ is called a stability ball of the line balance $b_{0} \in B(t)$, if for each vector $t^{\prime}=\left(\widetilde{t}^{\prime}, \bar{t}\right)$ of the processing times with

$$
\widetilde{t^{\prime}} \in O_{\rho}^{1}(\widetilde{t}) \bigcap R_{+}^{\tilde{n}}
$$

the line balance $b_{0}$ remains feasible and optimal. The maximal value $\rho_{b_{0}}^{1}(t)$ of the radius $\rho$ of a stability ball $O_{\rho}^{1}(\widetilde{t})$ is called the stability radius of the line balance $b_{0}$ for the problem SALBP-1.

The criterion (i.e., the necessary and sufficient condition) for the equality $\rho_{b_{0}}^{1}(t)=0$ and a general formula for calculating the stability radius $\rho_{b_{0}}^{1}(t)$ have been derived by Sotskov et al. (2006).

In the definition of the stability radius of an optimal line balance for the problem SALBP-1, used by Sotskov and Dolgui (2001), it is assumed that the open ball $O_{\rho}^{1}(\widetilde{t})$ is totally located within the space $R_{+}^{\tilde{n}}$. Therefore, in Sotskov and Dolgui (2001), generally smaller stability radii may be obtained. In particular, due to the definition of the stability radius of an optimal line balance used by Sotskov and Dolgui (2001), the stability radius of an optimal line balance cannot be grater than

$$
\min \left\{t_{i}: i \in \widetilde{V}\right\}
$$

So, Definition 2 is more appropriate for a practical use than that introduced by Sotskov and Dolgui (2001).

\section{CONCLUSION}

Along with using the lower bound on the value $\delta\left(b_{0}, b_{g}, t\right)$ given in Lemma 3, we can restrict further the number of line balances $b_{r} \in B \backslash\left\{b_{0}\right\}$ with which a comparison of the line balance $b_{0}$ has to be done for calculating the stability radius $\rho_{b_{0}}(t) \neq 0$.

In particular, one does not need to distinguish the line balances, which have different orders of the subsets in

$$
V=V_{1}^{b_{r}} \bigcup \ldots \bigcup V_{m}^{b_{r}}
$$

but their sets of subsets

$$
\left\{V_{1}^{b_{r}}, \ldots, V_{m}^{b_{r}}\right\}
$$

are the same.

Note also, that in practice not all line balances are suitable for a realization in the assembly line since not only precedence constraints defined by the set of $\operatorname{arcs} A$ have to be taken into account. Due to this, the cardinality of the set of line balances tested in Theorem 2 and in Lemma 3 may be considerably smaller than the cardinality of the set $B$.

It is easy to see that problem SALBP-2 with $n$ unordered operations $V$ (i.e., with digraph $G=(V, \emptyset))$ and $m$ working stations, may be interpreted as the problem of optimal scheduling $n$ unordered jobs on $m$ parallel identical machines with the makespan criterion. Therefore, the results presented in Sections 1-5 on the stability analysis of an optimal line balance for the problem SALBP-2 with $n$ unordered operations and $m$ working stations may be interpreted as new results on the stability analysis of a schedule minimizing the makespan for processing $n$ unordered jobs on $m$ parallel identical machines.

\section{REFERENCES}

H. Bräsel, Yu.N. Sotskov, F. Werner. Stability of a schedule minimizing mean flow time. Mathematical and Computer Modelling 24(10):39-53, 1996.

E. Erel, S.C. Sarin. A survey of the assembly line balancing procedures. Production Planning \& Control 9(5):414434, 1998.

A. Scholl. Balancing and Sequencing of Assembly Lines. Physica-Verlag, Heidelberg, Germany, 1999.

Yu.N. Sotskov, A. Dolgui. Stability radius of the optimal assembly line balance with fixed cycle time. In: IEEE Symposium on Emerging Technologies and Factory Automation, ETFA'2001. 1:623-628, 2001.

Yu.N. Sotskov, A. Dolgui, M.-C. Portmann. Stability analysis of an optimal balance for an assembly line with fixed cycle time. European Journal of Operational Research 168(3):783-797, 2006.

Yu.N. Sotskov, A. Dolgui, N. Sotskova, F. Werner. Stability of optimal line balance with given station set. In: Supply Chain Optimization, Applied Optimization. Springer, New York, USA. 94:135-149, 2005.

Yu.N. Sotskov, N. Sotskova, T.-C. Lai, F. Werner. Scheduling under Uncertainty. Theory and Algorithms. Belorusskaya nauka, Minsk, Belarus, 2010.

Yu.N. Sotskov, N. Sotskova, F. Werner. Stability of an optimal schedule in a job shop. Omega 25(4):397-414, 1997. 